Roughly speaking, a line striking northward from the head of the Bay of Bengal, to the Himalayas, then turning westward and skirting the southern flanks of that great mountain-chain, passing north of Cashmere, and in a westward direction to the Sea of Aral, the Caspian, and the Ural Mountains, divides the area occupied by people of each type, those to the east and north of this line being mainly Mongolians, and those to the south and west mainly Caucasians.

The people of India proper, except in the extreme north and north-east, belong mainly to the Caucasian division. It has been thought that other races have contributed a share to the composition of the present population of the Peninsula, having been the earliest inbabitants of the land, and forming, as it were, a substratum of the existing population; these are :-I. Negritos, allied to the Andaman Islanders: II. Australoids, allied to the modern Australians. The search for evidence for or against the existence of these elements in the population of India must naturally be sought for among the tribes which retain more or less of their barbarous condition.

With these must be classed the Veddahs of Ceylon. Many of these people have become civilised, but the wild or "Rock Veddahs" live in a most primitive state of social culture, without clothing, agriculture, or fixed dwellings. They are a dwarfish, stunted race, of blackish complexion. and with straight, though generally unkempt and shaggy hair. The condition of their bones and other physical characters give the jmpression of their being a race de - enerated and enfeebled by generations of privation and other circumstances adverse to full development. There are no Veddah skeletons in the College Museum, but as many as seven crania; one of them, that of a woman, is the smallest adult skull in the whole collection, its cerebral capacity measuring only 960 c.c. $(58.6$ c. ins.). They are all dolichocephalic, the average latitudinal index being $7 \mathrm{r}^{\circ} \mathrm{I}$, and the average altidudinal index $86^{\circ} \mathrm{I}$. Their prognathism is not very marked, and the nasal index is medium, averaging $50^{\circ} 3$. They enter rather into the type of the lower grades of the inhabitants of Bengal.

The present population of India, excluding the Mongoloid people of the north and east, is separated by language into two great divisions-(I) The Aryans, and (2) The Non-Aryans, the majority of whom speak one of the agglutinative tongues collectively called Dravidian. The Aryans came into India by the north-west by way of the Punjaub, about I, 500 years before our era, as is supposed. They now occupy the great alluvial plains of the Indus and Ganges between the Himalayas and the elevated plateau of Central India, and have spread southwards along both coasts as far as Ceylon.

The Dravidians, who occupy the greater part of the country southwards from the Nerbudda River, are supposed to be descended mainly from the people who inhabited the country before the Aryan immigration. They are again divided into two groups- $(a)$ The civilised Dravidians, speaking Tamul, Telugu, Canarese, \&c., and $(b)$ The wild tribes of the mountainous districts of the in. terior. These "Hill Tribes," as they are often called, are of great interest to the ethnologist, as they represent the oldest stratum of the population. By their languages they are divided into two groups--(I) Those that speak Dravidian, the Gonds, the Khonds, the Oraons, \&c.; (2) The Munda or Kolarian family, composed of numerous tribes called Coles, Hos, Moondahs, Santals, Billahs, \&c. Very little is known of the physical characters of these people.

Dr. John Shortt has lately sent to the Museum a series of twenty skulls belonging to the tribes of Maravars, who inhabit the Madura district in the south of the peninsula. He has also sent a series of equal number to the Museum of the Paris Anthropological Society, which has been described-by $M$. Callamand in a recent number of the
Rewue d'Anthropologie. As regards the capacity, the average in the Paris skulls is $I, 28 \mathrm{I}$ c.c., in those sent to the Museum, I, 268 c.c. The average lengths are respectively 174.5 and $175^{\circ} 6$, but in the former the projection of glabella is included. The average breadth is $13 \mathrm{I}$ in both; the latitudinal index is 746 in the one and $75 \mathrm{I}$ in the other series; the altitudinal, the nasal, and the orbital indices are respectively 752 and 758,521 and 510,839 , and 854 . These skulls, on the whole, differ totally from those of the Andamanese, especially in the great development of the occipital region. Nor do they present any striking resemblances either to those of the Australians, or to any of the Mongoloid races. Their characters do not differ much from those of the mixed population of Bengal generally. These Maravars may not belong to the races among which the characters of the original hill-tribes should be looked for, and no evidence has yet been found of cranial conformation bearing out the view of the Australian affinities of these people, derived from external appearance. The presence of a Negrito, i.e., woolly-haired and brachycephalic, element in the population of India, is also based at present on very slender evidence.

(To be continued)

\section{ON THE SECULAR EFFECTS OF TIDAL FRICTION ${ }^{1}$}

$\mathrm{T}$ three papers, read at different times before the Royal Society, the author has considered the theory of the tides of a viscous spheroid, and the perturbations of the rotation of the spheroid caused by the attraction of the tide-raising satellite; the direction of that investigation was governed by considerations of applicability to the case of the earth, moon, and sun.

In the paper, of which we are here giving an account, the question is considered both from a more general and from a more special point of view than in the previous papers. For it is here supposed that there is only a single tide-raising body or satellite which moves in a circular orbit in the equator of the planet, but the orbital motion may be either consentaneous with or adverse to the planet's rotation. The tides supposed to be raised in the planet by the attraction of the satellite are of any kind whatever, provided that there is a frictional resistance in the planet to the tidal motion. The results are therefore applicable alike to the hypothesis of bodily tides, or to that of oceanic tides.

It results from a general mechanical principle that in whatever way the satellite and planet interact, the whole moment of momentum of the rotation of the system must remain constant; whilst, as there is a frictional resistance in the planet to the tidal motion, the whole energy of the system, viz., the sum of the potential and kinetic energies, must diminish. The method employed to trace the effects of tidal friction consists in drawing two curves, one of which represents the constancy of the moment of momentum, and the other of which gives the energy of the system for each configuration.

Then if we conceive a system of a planet and satellite started in such a way as to be represented by a given point on the curve of conservation of moment of momentum; and if we imagine this point linked to its corresponding point on the curve of energy, since the energy must degrade, the point on the curve of energy must always slide down a slope and carry with it the point on the curve of momentum.

It is thus possible to track the nature of the changes in the configuration of the system, but the method gives no clue to the time occupied by those changes. This comparison of the energy with the moment of momentum of the system by a graphical method was suggested to the author by Sir William Thomson.

I A paper read before the Royal Cociety on Iure 19, I879, by G. IFo Darwin. 
A third curve is also introduced which represents such a rotation of the system that the planet always shows the same face to the satellite, just as we always see the same face of the moon; this curve is called the curve of rigidity, because when the motion is of this kind, the system moves as though the planet and satellite were parts of one rigid body.

It appears from a consideration of these three curves that if the whole momentum of the system be less than a certain amount, then it is not possible to set a given planet and satellite in rotation, so that the planet shall always show the same face to the satellite; but if this can be done at all, it can be done in two ways, and one of those ways corresponds to a maximum amount of energy of the system, and the other to a minimum. Moreover the configuration of maximum energy is one of dynanical instability and the system may degrade in either of two ways from that state. In one of these modes of degradation the satellite approaches and falls into the planet, and in the other it recedes from the planet.

Part of the author's previous papers consists in tracing backwards the moon's motion from its present conditicn to that configuration of maximum energy; and it was found that that state corresponded with a rotation of the earth and moon, like the parts of one rigid body, in about five hours. This rapid periodic time of the moon corresponds with only a few thousands of miles intervening between the earth's surface and that body. Since the tides on the earth must be subject to friction, it follows inevitably that, if time enough has elapsed since the origin of the moon and earth, the present state must be a degradation of the configuration of maximum energy, which cannot itself be a degradation of a previous state. And therefore it was maintained that this closeness of the two bodies points to the community of their origin.

In this mode of evolution we see that the rotation and revolution of the two bodies was primitively such that the month and day were of equal length (about five hours), and that in the future they will again come to equality, each being then about fifty of our days long. From this it follows that the system must pass through some phase in which there is a maximum number of planetary rotations during one revolution of the satellite, or shortly there must, at some time, be a maximum number of days in the month. Numerical calculation shows that for the earth and moon that maximum number is about 29, and that at present, when we have $27 \frac{1}{3}$ days in the sidereal month, we have slightly passed that maximum.

From a further consideration of the figures it appears that if the planet and satellite are set in motion with opposite rotations, the satellite will fall into the planet if the moment of momentum of orbital motion be less than or equal to, or only greater, by a certain critical amount than the moment of momentum of planetary rotation; but if it be greater by more than a certain critical amount the satellite will approach the planet, the rotation of the planet will stop and reverse, and finally the system will come to equilibrium when the two bodies move round as a rigid body, with a long periodic time.

If the rate of the planet's rotation be less than that of the satellite's revolution, so that the sidereal month is shorter than the day (as with the inner satellite of Mars), then the satelite will either approach the planet and ultimately fall into it, or will approach the planet and will finally move round the planet at the same rate as the planet rotates. It depends on the nature of the system, as to which of these two cases will be the result.

The method is then extended to the case where the satellite, instead of being merely an attractive particle, is also a spheroid rotating about an axis perpendicular to the plane of the orbit. In this case the graphical illustration is by means of surfaces, there being one surface representing conservation of moment of momentum, and another representing the energy of the system. Each point on one surface has a corresponding point on the other surface, and the point on the energy surface must always slide down hill. It is not necessarily the case that the descent should be down a line of greatest slope. Illustrations are given to show that a point on an energy surface may sometimes depart from the bottom of a furrow, or may descend a ridge on the surface. The path to be followed by the point on the energy surface depends on the nature of the tides raised in the two bodies. Thus the solution in this case is not determinate, without some further knowledge of the system.

\section{MORE NOTES FROM KILBURN}

$T H E$ gas and petroleum-engines make a fair show. The Otto silent gas-engine, however, seems still to hold its own, other constructions having, so far as one can judge from a short inspection at Kilburn, some defect or other, such as noisy working, or a dangerouslooking outside flare of the ignited gas. One striking novelty is shown in this section, namely, an invention of Mr. Iugald Clerk, whereby the gaseous mixture is. lighted by a cage of platinum wire, which retains heat enough from one ignition to the next to be effective for this purpose. It is stated that 400 ignitions have been made in the cylinder of an engine of this type in one minute. There are other distinctive contrivances in this gas-engine, which, by the by, is the manufacture of Messrs. Thomson, Sterne, and Co., and altogether show.s great ingenuity. One of the engines exhibited by this frrm is described as a "Domestic Motor," of $\frac{3}{10}$ horsepower, costing but $15 l$, and deriving its power from steam generated by the ignition of air and gas. Such an engine seems admirably fitted for private use in laboratories and small workshops as well as in houses.

Self-binding harvesters, in which wire is used for the automatic tying-up of the sheaves, are attracting much attention, now that millers have ajopted the use of magnets, preferably electro-magnets, to separate any bits of iron wire that may happen to get mixed with the grain.

The machinery and implements employed in buttermaking and cheese-making afford a striking example of the advantages resulting from the application of scientific exactness to a most useful art. The question of temperature in every operation of the dairy is now recognised as of extreme importance. The thermometer reigns supreme in the interesting tent where dairying is shown. The material, the depth, and the diameter of the pans for "setting" milk have been duly studied. Though the processes and instruments exhibited in action are few in comparison with those at the previous shows at Hamburg and other special gatherings, yet the exhibits of Mr. E. Ahlborn and of the Aylesbury Dairy Company are worth attentive study. We noted especially an ingenious butter-squeezer or presser, which removes in the most effectual way that very variable and often excessive quantity of butter-milk and water and of interstitial air which occur in ordinary butter, even when quite genuine. Not only is liability to change and decomposition much lessened by this operation, but constancy of composition is secured, and we no longer find the proportion of water in fresh butter ranging between 6 and 20 per cent.

Messrs. F. H. Atkins and Co. show some good models and examples of their water-filters and other sanitary appliances in connection with water supply, One of their contrivances is specially clever. The surfaces of filtering media of course become rapidly clogged and useless when media of course become rapidly clogged The vertical surfaces of Atkins's cloth filters are so arranged as to be capable of rotation against a rotating cylindrical brush, set vertically, and accommodating itself perfectly to the surface to be cleansed.

An instructive section of the Killurn Show is that deroted to hops. From a paper issued by Messrs. John 